\title{
Propofol ou sevofluorano sobre variáveis hemodinâmicas em cães submetidos à administração subaracnóidea de iohexol
}

\author{
Propofol or sevoflurane upon hemodynamic variables in dogs submitted \\ to subarachnoid administration of iohexol
}

\section{Celina Tie Nishimori ${ }^{1}$ Newton Nunes ${ }^{2}$ Alessandra Valeiro Leite ${ }^{1}$ Danielli Parrilha de Paula ${ }^{1}$ Márlis Langenegger de Rezende ${ }^{3}$ Almir Pereira de Souza ${ }^{4}$ Paulo Sérgio Patto dos Santos ${ }^{1}$}

\section{RESUMO}

Avaliaram-se os efeitos do propofol ou sevofluorano sobre variáveis hemodinâmicas, em cães submetidos à mielografia. Para tanto, utilizaram-se trinta animais distribuídos em dois grupos de igual número, denominados GP e GS. Os animais do GP receberam propofol $\left(10 \mathrm{mg} \mathrm{kg}^{-1}\right)$ por via intravenosa para intubação orotraqueal, $e$, imediatamente após, administrou-se continuamente, por meio de bomba de infusão, propofol $\left(0,55 \pm 0,15 \mathrm{mg} \mathrm{kg}^{-1}\right.$ $\mathrm{min}^{-1}$ ). Decorridos 30 minutos, foi colhido liquor na cisterna magna e o meio de contraste iohexol foi injetado. No GS empregou-se a mesma metodologia adotada para o GP, utilizando indução anestésica pela administração de sevofluorano a 2,5 CAM, com o uso de máscara naso-oral vedada e manutenção com 1,5 CAM. As variáveis estudadas foram freqüência cardíaca (FC), pressões arteriais sistólica, diastólica e média (PAS, PAD e PAM, respectivamente), débito cardíaco (DC), volume sistólico (VS), pressão venosa central $(P V C)$, freqüência respiratória $(f)$, concentração de dióxido de carbono ao final da expiração $\left(E^{2} \mathrm{CO}_{2}\right)$ e saturação de oxihemoglobina $\left(\mathrm{SpO}_{2}\right)$. As mensurações das variáveis foram realizadas imediatamente após a colheita do liquor (M1), logo após a aplicação de iohexol (M2), seguida das demais em intervalos de 10 minutos, durante uma hora. Utilizazouse a Análise de Variância (ANOVA) seguida pelo teste F como métodos estatísticos, considerando $P<0,05$. Houve redução da pressão arterial em ambos os grupos, sendo menos intensa no grupo que recebeu propofol. No GS, observou-se redução da FC e aumento do DC e do VS. Referente a $\mathrm{ETCO}_{2}$ as médias do GP foram superiores às do GS. Os resultados obtidos permitiram concluir que tanto o propofol como o sevofluorano não promovem alterações hemodinâmicas que comprometam a técnica de mielografia.

\author{
Palavras-chave: anestesia, caninos, hemodinâmica, \\ mielografia.
}

\begin{abstract}
The effects of propofol or sevoflurane upon hemodynamics variables were studied in dogs submitted to myelography. For this purpose, thirty dogs were randomly divided into two equal groups, denominated GP and GS. The animals of GP were induced with propofol $\left(10 \mathrm{mg} \mathrm{kg}^{-1}\right)$ intravenously, intubated and after that, propofol was administrated continuously through an infusion pump $\left(0.55 \pm 0.15 \mathrm{mg} \mathrm{kg}^{-1} \mathrm{~min}^{-1}\right)$. After thirty minutes the liquor was collected on the magna cistern and the contrast (iohexol) was injected. To the animals of GS, the same methodology was adopted, but sevoflurane at 2.5 MAC was used as the anesthetic induction by facial mask, and the maintenance was done with 1.5 MAC. The variables studied were heart rate (HR), systolic, diastolic and mean arterial pressures (SAP, DAP, and MAP, respectively), cardiac output (CO), stroke volume (SV), central venous pressure (CVP), respiratory rate $(R R)$, end tidal carbon dioxide $\left(\mathrm{ETCO}_{2}\right)$ and oxyhemoglobin saturation $\left(\mathrm{SpO}_{2}\right)$. The measurements were realized immediately after liquor collection (M1), after iohexol administration (M2), and at each 10 minutes after M2, during one hour. The numerical data were submitted to Analysis of Variance (ANOVA) followed by $F$ test considering $P<0.05$. There was reduction in blood pressure at both groups, and this effect was less intense at the group that received propofol. In GS it was observed reduction in HR and increase in CO and SV. Regarding to $\mathrm{ETCO}_{2}$ the averages of GP were greater than the GS. The results allow to conclude that both propofol and sevoflurane do not promote hemodynamics alterations that compromise the myelography technique.
\end{abstract}

Key words: anesthesia, canine, hemodynamic, myelography.

\footnotetext{
${ }^{1}$ Faculdade de Ciências Agrárias e Veterinárias (FCAV), Universidade Estadual Paulista (UNESP), Campus de Jaboticabal, Jaboticabal, SP, Brasil.

${ }^{2}$ Departamento de Clínica e Cirurgia Veterinária, FCAV, UNESP, Campus de Jaboticabal. Via de Acesso: Prof. Paulo Donato Castellane s/n, Zona rural, 14884-900, Jaboticabal, SP, Brasil. E-mail: newton@fcav.unesp.br. Autor para correspondência.

${ }^{3}$ Universidade da Califórnia, Davis, CA, EUA.

${ }^{4}$ Universidade Federal de Campina Grande, Campus de Patos, Patos, PB, Brasil.
} 


\section{INTRODUÇÃO}

Em Medicina Veterinária a mielografia é um procedimento diagnóstico de contraste bastante utilizado e útil na neurorradiologia devido, principalmente, ao baixo custo quando comparado aos equipamentos necessários para as técnicas não invasivas. Para a realização desse exame, são necessários a anestesia geral por via intravenosa ou inalatória, monitoração constante e suporte ventilatório adequado (LEITE et al., 2002).

O propofol (2,6 diisopropilfenol) é um agente hipnótico intravenoso de curta ação (QUANDT et al., 1998) que pode ser usado como agente de indução e também manutenção anestésica na forma de bolus intermitentes ou infusão contínua (DENEUCHE \& DESBOIS, 1999). Proporciona indução rápida e sem sinais de excitação (GLOWASKI \& WETMORE, 1999).

Sobre o sistema cardiovascular, o emprego do propofol está associado à discreta diminuição na pressão arterial (SHORT \& BUFALARI, 1999). Essa ação depressora do fármaco está relacionada a efeitos depressores diretos sobre o miocárdio e a vasodilatação arterial e venosa (FANTONI, 2002). A redução na pressão arterial é proporcional ao aumento da concentração plasmática do agente anestésico (WHITWAM et al., 2000). Foi relatada por KEEGAN \& GREENE (1993) a redução da freqüência cardíaca durante anestesia com propofol, caracterizando também o efeito cronotrópico negativo (QUANDT et al., 1998). No entanto, AGUIAR et al. (1993) observaram o aumento da freqüência cardíaca, especialmente após 20 minutos de anestesia, coincidindo com a diminuição dos valores da pressão arterial. Segundo FANTONI (2002), tanto taquicardia quanto bradicardia podem ser verificadas com esse agente.

O propofol pode causar depressão respiratória e apnéia após a sua administração, principalmente em injeções rápidas (MUIR III \& GADAWSKI, 1998; QUANDT et al., 1998), expressa por redução do volume corrente e da freqüência respiratória (SHORT \& BUFALARI, 1999). Dessa forma, é desejável que a aplicação seja realizada em torno de 90 a 120 segundos, na tentativa de minimizar essas ocorrências (QUANDT et al., 1998; CORTOPASSI, 2002). KEEGAN \& GREENE (1993) recomendaram suporte ventilatório durante a infusão contínua de propofol.

O sevofluorano é um éter isopropílico fluoretado com concentração alveolar mínima (CAM) de 2,36 V\%, em cães. O seu baixo coeficiente de solubilidade sangüínea $(0,68)$ lhe confere rápida indução e recuperação anestésica com irritação mínima das vias aéreas superiores, baixa incidência de tosse e laringoespasmo, rápido controle da profundidade anestésica e preservação da ventilação espontânea (CHARLES \& FALLON, 2000).

Promove efeito inotrópico negativo sobre o sistema cardiovascular (HANOUZ et al., 2000), aumentando o fluxo sangüíneo coronariano e diminuindo o consumo de oxigênio pelo miocárdio (CRYSTAL et al., 2000), além de provocar redução da pressão arterial sangüínea e da resistência vascular sistêmica (BRANSON et al., 2001), todos dosedependente. À semelhança do isofluorano, o sevofluorano não sensibiliza o coração às catecolaminas (OMOIGUI, 1998). Dentre os anestésicos voláteis, parece ser o que oferece maior estabilidade cardiovascular (EBERT et al., 1995).

A exemplo de outros agentes anestésicos inalatórios, o sevofluorano produz depressão respiratória dose-dependente (BRANSON et al., 2001; OLIVA, 2002), agindo diretamente sobre o centro ventilatório medular e indiretamente sobre os músculos intercostais (OMOIGUI, 1998). No entanto, a depressão respiratória é considerada baixa (TACKE et al., 1998), mesmo utilizando anestesia em baixo fluxo (MUIR III \& GADAWSKI, 1998).

Iohexol é um meio de contraste não iônico, solúvel em água, usado para mielografias em cães (LEITE et al., 2002). Concentrações de iohexol da ordem de 180 a 350 $\mathrm{mg} \mathrm{mL}^{-1}$ são as mais utilizadas (SIMON \& NICHOLAS, 1999). LEWIS \& HOSGOOD (1992) preconizaram concentrações de iohexol de 240mg $\mathrm{mL}^{-1}$ e doses de 0,3 a $0,5 \mathrm{~mL} \mathrm{~kg}^{-1}$. Comparativamente, o iohexol e o meio de contraste iopamidol não apresentam diferenças na qualidade das imagens produzidas ou nas reações adversas (WIDMER et al., 1992), sendo portanto, os mais empregados na Medicina Veterinária.

Em outro estudo, LEWIS \& HOSGOOD (1992) compararam os efeitos dos meios de constraste metrizamide e iohexol e observaram menor incidência de convulsões após o uso deste último.

Devido aos riscos inerentes à técnica radiográfica da mielografia e a escassez de informações relativas às alterações na hemodinâmica promovidas pelo propofol e sevofluorano, em cães submetidos a esta técnica radiográfica, objetivou-se avaliar as possíveis alterações dessas variáveis em cães submetidos à administração subaracnóidea de iohexol.

\section{MATERIAL E MÉTODOS}

Foram utilizados 30 cães, sem raça definida, clinicamente saudáveis, com peso corpóreo variando 
de 6,5 a 16,5kg, fornecidos pelo canil do Hospital Veterinário da UNESP, Câmpus de Jaboticabal, distribuídos aleatoriamente em dois grupos de igual número denominados GP e GS. Os animais foram submetidos a jejum alimentar de 12 horas e restrição hídrica de duas horas.

Para os animais do GP, induziu-se à anestesia geral por meio da administração intravenosa de propofol $^{\mathrm{a}}$, na dose de $10,0 \pm 0,5 \mathrm{mg} \mathrm{kg}^{-1}$. Após intubados com sonda de Magill, receberam oxigênio a 100\% em fluxo de $30 \mathrm{~mL} \mathrm{~kg}^{-1}$ minuto $^{-1}$ através de circuito anestésico com reinalação parcial de gases $^{\mathrm{b}}$ e administrou-se continuamente pela via intravenosa, por meio de bomba de infusão ${ }^{c}$, propofol na dose de $0,55 \pm$ $0,15 \mathrm{mg} \mathrm{kg}^{-1}$ minuto $^{-1}$. Os cães foram mantidos em decúbito lateral esquerdo, com a cabeça elevada em ângulo de $30^{\circ}$ em relação ao plano horizontal da mesa. Decorridos trinta minutos, foi feita a colheita de líquido cérebro espinhal (LCE) na cisterna magna, na quantidade de $0,3 \mathrm{~mL} \mathrm{~kg}^{-1}$, usando-se uma agulha estéril. Ato contínuo, uma seringa com o meio de contraste iohexol ${ }^{\mathrm{d}}$ a $30 \%$, no volume de $0,3 \mathrm{~mL} \mathrm{~kg}^{-1}$, foi acoplada na agulha e o conteúdo injetado lentamente, durante 90 segundos.

Nos animais do GS, induziu-se a anestesia geral com sevofluorano a 2,5 CAM, mensurada em analisador de gases ${ }^{\mathrm{f}}$. $\mathrm{O}$ anestésico foi fornecido diluído em fluxo de oxigênio de $150 \mathrm{~mL} \mathrm{~kg}^{-1}$ minuto $^{-1}$, com o uso de máscara naso-oral vedada e circuito anestésico com reinalação parcial de gases, durante o tempo necessário para permitir a intubação orotraqueal. Após intubados com sonda de Magill, o oxigênio foi ajustado para um fluxo de $30 \mathrm{~mL} \mathrm{~kg}^{-1}$ minuto ${ }^{-1}$ e o vaporizador para 1,5 CAM. Em seguida, aplicou-se procedimento similar ao realizado no grupo GP, para a administração do contraste.

As avaliações dos parâmetros foram realizadas imediatamente antes da coleta do LCE (M1), após a coleta do LCE e administração de iohexol (M2) e a cada 10 minutos, durante 60 minutos, totalizando oito mensurações.

Os parâmetros mensurados foram a freqüência cardíaca (FC), por meio de eletrocardiógrafo computadorizado ${ }^{\text {, }}$ pressões arteriais sistólica, diastólica e média (PAS, PAD e PAM, respectivamente), utilizando-se monitor multiparamétrico ${ }^{\mathrm{h}}$ cujo sensor foi adaptado em cateter, introduzido na artéria femoral esquerda.

O débito cardíaco (DC), o volume sistólico (VS) e a pressão venosa central (PVC) foram mensurados com o uso de dispositivo microprocessado ${ }^{\mathrm{i}}$ para medida direta, empregando-se cateter de Swan-Ganz, introduzido cirurgicamente na veia femoral esquerda.
A freqüência respiratória $(f)$ e concentração de dióxido de carbono ao final da expiração $\left(\mathrm{ETCO}_{2}\right)$ foram obtidas por leitura direta em monitor multiparamétrico ${ }^{\mathrm{f}}$, cujo sensor foi conectado entre a sonda orotraqueal e o equipamento de anestesia e a saturação de oxihemoglobina $\left(\mathrm{SpO}_{2}\right)$ foi obtida por leitura direta em monitor multiparamétrico ${ }^{\mathrm{f}}$, sendo o emissor/sensor adaptado na língua dos animais.

A avaliação estatística das variáveis analisadas foram efetuadas por meio de Análise de Variância (ANOVA) seguida pelo teste F, considerando-se um nível de $5 \%$ de probabilidade.

Este trabalho foi aprovado pela Comissão de Ética e Bem Estar Animal da FCAVJ/Unesp e, terminado o estudo, os animais foram oferecidos para adoção.

\section{RESULTADOS E DISCUSSÃO}

A literatura consultada é unânime ao afirmar que o sevofluorano promove redução da pressão arterial de modo dose-dependente (MUTOH et al., 1997; BRANSON et al., 2001; OLIVA, 2002). Observouse discreto aumento da PAM no GS em M2, entretanto, esta alteração não foi sustentada nos demais momentos, provavelmente devido à diminuição da resistência vascular sistêmica promovida pelo fármaco (Tabela 1). A PAS e a PAD apresentaram comportamento similar, mostrando íntima relação com a PAM.

O propofol também apresenta ação depressora na pressão arterial, relacionada a efeitos diretos sobre o miocárdio e a vasodilatação arterial e venosa (SHORT \& BUFALARI, 1999). Porém, ao observar a tabela 1 , nota-se que sua ação na pressão arterial foi menos intensa que a provocada pelo sevofluorano. A análise estatística demonstrou que a PAM no GP, ao contrário do GS, apresentou aumento em M2 que permaneceu constante até M6, indicando que a retirada do LCE e administração do contraste não proporcionaram variações dignas de nota.

O DC é determinado pela FC e pelo VS portanto, qualquer fator que altere a freqüência do batimento cardíaco ou o volume de ejeção pode modificar seus valores (ALMEIDA et al., 2000). Apesar de ter sido observada no GS a redução da FC e sabendo-se que os valores do DC neste mesmo grupo apresentaram aumento, pode-se afirmar que a elevação do VS foi determinante na obtenção do achado (Tabela 1). O aumento do DC pode estar intimamente relacionado à redução da resistência vascular sistêmica, promovida pelo anestésico volátil, pois sabe-se que a redução nos valores de pós-carga 
Tabela 1 - Valores médios e desvios padrão $(\mathrm{x} \pm \mathrm{s})$ das variáveis freqüência cardíaca (FC), pressões arteriais sistólica (PAS), diastólica (PAD) e média (PAM), débito cardíaco (DC), volume sistólico (VS), pressão venosa central (PVC), freqüência respiratória (f), concentração de dióxido de carbono expirado $\left(\mathrm{ETCO}_{2}\right)$ e saturação de oxihemoglobina $\left(\mathrm{SpO}_{2}\right)$, obtidos em cães anestesiados pelo propofol ou sevofluorano e submetidos a administração subaracnóidea de iohexol.

\begin{tabular}{|c|c|c|c|c|c|c|c|c|c|}
\hline VARIÁVEIS & & M1 & M2 & M3 & M4 & M5 & M6 & M7 & M8 \\
\hline FC & GS & $122 \pm 17^{\mathrm{a}}$ & $117 \pm 21^{\mathrm{ab}}$ & $113 \pm 21^{b}$ & $115 \pm 19^{b}$ & $113 \pm 18^{b}$ & $113 \pm 17^{\mathrm{b}}$ & $112 \pm 18^{b}$ & $113 \pm 18^{\mathrm{b}}$ \\
\hline (bat./min.) & GP & $119 \pm 16^{\mathrm{ab}}$ & $122 \pm 14^{\mathrm{a}}$ & $123 \pm 18^{\mathrm{a}}$ & $119 \pm 17^{\mathrm{a}}$ & $115 \pm 16^{\mathrm{b}}$ & $118 \pm 20^{\mathrm{ab}}$ & $118 \pm 20^{\mathrm{ab}}$ & $116 \pm 20^{\mathrm{ab}}$ \\
\hline PAS & GS & $115 \pm 12^{* a b c}$ & $121 \pm 18^{*^{\mathrm{a}}}$ & $111 \pm 21 * \mathrm{abc}$ & $113 \pm 16^{* \mathrm{~b}}$ & $110 \pm 14^{* a b c}$ & $109 \pm 15^{* \mathrm{c}}$ & $110 \pm 15^{* a b c}$ & $111 \pm 15^{* a b c}$ \\
\hline (mmHg) & GP & $132 \pm 22^{\mathrm{a}}$ & $145 \pm 24^{\mathrm{b}}$ & $144 \pm 21^{\mathrm{b}}$ & $142 \pm 20^{\mathrm{b}}$ & $143 \pm 20^{\mathrm{b}}$ & $142 \pm 17^{\mathrm{b}}$ & $143 \pm 17^{\mathrm{b}}$ & $143 \pm 17^{\mathrm{b}}$ \\
\hline PAD & GS & $67 \pm 13^{*^{\mathrm{a}}}$ & $72 \pm 19 *^{\mathrm{a}}$ & $58 \pm 18^{* \mathrm{~b}}$ & $58 \pm 12 *^{\mathrm{b}}$ & $57 \pm 12^{*^{\mathrm{b}}}$ & $56 \pm 12^{* \mathrm{~b}}$ & $56 \pm 12^{* \mathrm{~b}}$ & $55 \pm 13^{* b}$ \\
\hline (mmHg) & GP & $79 \pm 15^{\mathrm{a}}$ & $90 \pm 18^{\mathrm{b}}$ & $89 \pm 16^{\mathrm{b}}$ & $86 \pm 18^{\mathrm{ab}}$ & $85 \pm 18^{\mathrm{ab}}$ & $84 \pm 17^{\mathrm{ab}}$ & $84 \pm 14^{\mathrm{ab}}$ & $83 \pm 14^{\mathrm{ab}}$ \\
\hline PAM & GS & $86 \pm 12^{* a c}$ & $90 \pm 18 *^{\mathrm{c}}$ & $79 \pm 19 *$ ab & $80 \pm 13^{* a b}$ & $78 \pm 12^{* a b}$ & $78 \pm 12 *$ b & $78 \pm 13^{* a \mathrm{~b}}$ & $78 \pm 11^{* \mathrm{~b}}$ \\
\hline (mmHg) & GP & $99 \pm 17^{\mathrm{a}}$ & $113 \pm 18^{b}$ & $110 \pm 17^{\mathrm{b}}$ & $108 \pm 19^{\mathrm{b}}$ & $108 \pm 17^{\mathrm{b}}$ & $107 \pm 15^{b}$ & $106 \pm 13^{\mathrm{ab}}$ & $107 \pm 14^{\mathrm{ab}}$ \\
\hline $\mathrm{DC}$ & GS & $2,3 \pm 0,6^{\mathrm{a}}$ & $2,4 \pm 0,7^{\mathrm{a}}$ & $2,5 \pm 0,7^{\mathrm{a}}$ & $2,6 \pm 0,7^{\mathrm{b}}$ & $2,6 \pm 0,7^{\mathrm{ab}}$ & $2,6 \pm 0,8^{b}$ & $2,6 \pm 0,8^{\mathrm{b}}$ & $2,7 \pm 0,9^{b}$ \\
\hline (L/min.) & GP & $2,6 \pm 0,8$ & $2,7 \pm 1,3$ & $2,5 \pm 1,1$ & $2,5 \pm 1,1$ & $2,5 \pm 1,1$ & $2,5 \pm 1,1$ & $2,5 \pm 1,0$ & $2,5 \pm 1,1$ \\
\hline VS & GS & $18,9 \pm 4,9^{\mathrm{a}}$ & $20,8 \pm 5,9^{a}$ & $22,2 \pm 5,2^{b}$ & $22,7 \pm 5,7^{\mathrm{b}}$ & $23,2 \pm 6,3^{b}$ & $22,7 \pm 5,6^{b}$ & $23,1 \pm 5,4^{b}$ & $24,0 \pm 6,5^{b}$ \\
\hline$(\mathrm{mL})$ & GP & $20,8 \pm 7,5$ & $22,0 \pm 9,9$ & $20,7 \pm 8,2$ & $21,5 \pm 8,9$ & $22,3 \pm 8,4$ & $21,2 \pm 8,9$ & $20,9 \pm 8,0$ & $21,5 \pm 8,6$ \\
\hline PVC & GS & $2,1 \pm 1,6^{\mathrm{a}}$ & $2,7 \pm 1,9^{\mathrm{ab}}$ & $2,9 \pm 2,2^{\mathrm{ab}}$ & $2,5 \pm 1,8^{\mathrm{ab}}$ & $2,8 \pm 1,9^{\mathrm{ab}}$ & $3,1 \pm 2,0^{b}$ & $3,3 \pm 2,3^{b}$ & $2,9 \pm 2,3^{\mathrm{ab}}$ \\
\hline (mmHg) & GP & $3,0 \pm 1,8$ & $2,9 \pm 2,2$ & $2,3 \pm 2,0$ & $2,4 \pm 2,2$ & $2,9 \pm 2,1$ & $2,3 \pm 2,5$ & $2,9 \pm 2,8$ & $3,0 \pm 3,4$ \\
\hline$f$ & GS & $11 \pm 6 *$ & $9 \pm 5$ & $11 \pm 5$ & $12 \pm 7$ & $11 \pm 5$ & $11 \pm 5$ & $11 \pm 6$ & $11 \pm 6,82$ \\
\hline (mov./min.) & GP & $6 \pm 4^{\mathrm{a}}$ & $7 \pm 4^{\mathrm{a}}$ & $8 \pm 6^{\mathrm{a}}$ & $9 \pm 7^{\mathrm{a}}$ & $9 \pm 9^{\mathrm{b}}$ & $8 \pm 6^{\mathrm{a}}$ & $7 \pm 6^{\mathrm{a}}$ & $8 \pm 7,86^{\mathrm{a}}$ \\
\hline $\mathrm{ETCO}_{2}$ & GS & $43 \pm 5^{*^{\mathrm{a}}}$ & $36 \pm 15^{*^{a}}$ & $48 \pm 11 *^{\mathrm{b}}$ & $49 \pm 12^{\mathrm{ab}}$ & $48 \pm 13^{*^{\mathrm{ab}}}$ & $49 \pm 15^{\mathrm{ab}}$ & $44 \pm 18^{* \mathrm{ab}}$ & $49 \pm 17^{\mathrm{ab}}$ \\
\hline (mmHg) & GP & $58 \pm 11$ & $59 \pm 11$ & $57 \pm 11$ & $57 \pm 15$ & $59 \pm 16$ & $59 \pm 15$ & $58 \pm 16$ & $58 \pm 15$ \\
\hline $\mathrm{SpO}_{2}$ & GS & $100 \pm 0 *$ & $100 \pm 0$ & $100 \pm 0 *$ & $100 \pm 0$ & $100 \pm 0$ & $100 \pm 0$ & $100 \pm 0$ & $100 \pm 0 *$ \\
\hline$(\%)$ & GP & $99,6 \pm 0,7$ & $99,3 \pm 1,4$ & $99,5 \pm 0,7$ & $99,6 \pm 0,8$ & $99,5 \pm 1,1$ & $99,5 \pm 1,1$ & $99,5 \pm 1,0$ & $99,4 \pm 1,0$ \\
\hline
\end{tabular}

* Existe diferença estatística entre os grupos para este momento pelo teste $\mathrm{F}(\mathrm{p}<0,05)$.

Médias seguidas por letras diferentes, nas linhas, diferem significativamente entre si pelo teste $\mathrm{F}(\mathrm{p}<0,05)$

aumenta o volume ejetado pelos ventrículos (BRANSON et al., 2001).

Um dos melhores parâmetros na avaliação da volemia é a PVC, pois ela afeta a pré-carga, a qual é amplamente dependente do volume sangüíneo (CORTOPASSI, 2002). Uma vez que a PVC não apresentou alterações significativas entre os momentos, o aumento do VS no GS (Tabela 1) pode ser atribuído a uma discreta redução na pós-carga, possivelmente proveniente da diminuição da resistência vascular sistêmica gerada pelo sevofluorano, como já descrito e conforme relatado por BRANSON et al. (2001).

Apesar de FANTONI (2002) relatar que o propofol promove diminuição da PA e diminuição do DC ocasionada pela redução da pré-carga, nenhuma alteração foi observada nos parâmetros DC e VS em GP, apenas a FC aumentou em M2, M3 e M4 (Tabela 1).

Os valores de $\mathrm{ETCO}_{2}$ refletem de maneira muito próxima a pressão parcial arterial de $\mathrm{CO}_{2}\left(\mathrm{PaCO}_{2}\right)$, dando uma noção da qualidade da ventilação (NUNES, 2002). $\mathrm{A} \mathrm{PaCO}_{2}$ é um importante regulador do tônus da vasculatura cerebral, promovendo a vasodilatação sob condições de hipercapnia e a vasoconstrição sob condições de hipocapnia (KAWATA et al., 2001). Os animais do GP apresentaram valores de $\mathrm{ETCO}_{2}$ superiores aos do GS (Tabela 1). Segundo CHARLES \& FALLON (2000), a auto-regulação cerebral é melhor preservada sob condições de hipercapnia (50 a $66 \mathrm{mmHg}$ ) em humanos anestesiados com propofol do que com sevofluorano. No presente trabalho, pôde-se observar fato semelhante nos cães anestesiados com infusão contínua de propofol.

Depressão respiratória e apnéia durante a injeção de contraste podem acontecer quando a aplicação é muito rápida (ADAMS, 1982) ou pode ser decorrência da lesão na junção bulbo-medular durante a introdução da agulha para a realização da mielografia cervical (SIMON \& NICHOLAS, 1999). No GS, não foram observadas alterações na variável $f$ (Tabela 1 ). $\mathrm{O}$ aumento do $\mathrm{ETCO}_{2}$ pode ser considerado irrelevante, do ponto de vista biológico, uma vez que se manteve dentro dos parâmetros aceitáveis para a espécie. Também no GP, não foram observadas alterações de importância em relação à $f$, ou seja, ambos os fármacos mantiveram a ventilação espontânea e proporcionaram segurança durante o procedimento de mielografia.

Ciência Rural, v.35, n.6, nov-dez, 2005. 
A oximetria reflete a porcentagem de oxihemoglobina saturada por oxigênio, determinando alto grau de previsibilidade da hipoxia tissular, o que permite redução no índice de acidentes anestésicos relacionados à hipoxemia (NUNES, 2002). Não houve diferença significativa, em relação à $\mathrm{SpO}_{2}$, em todos os momentos e em ambos os grupos (Tabela 1). Tal achado demonstra que os fármacos estudados não foram capazes de promover alterações importantes neste parâmetro e, por isso, parecem não determinar risco de hipoxia durante mielografia.

Entretanto, ao se observarem os valores de oximetria na tabela 1 , nota-se que os valores do GS mantiveram-se sempre acima dos valores obtidos no GP. Ao se cotejarem esses valores com aqueles do VS e PAM, pode-se deduzir que a menor resistência vascular periférica produzida pelo sevofluorano, aliada ao discreto aumento do volume sistólico, permitiu que uma quantidade maior de sangue irrigasse tanto os sistemas de resistência como os de capacitância periféricos. Sabendo-se que o sensor do oxímetro foi posicionado segundo o citado por NUNES (2002), optando-se por região corpórea periférica, pode-se deduzir que os efeitos hemodinâmicos periféricos produzidos pelo anestésico volátil influenciaram positivamente na sensibilidade do equipamento utilizado para a mensuração.

\section{CONCLUSÕES}

Os resultados obtidos permitem concluir que o propofol e o sevofluorano não promovem alterações hemodinâmicas que comprometam a técnica de mielografia, sendo que ambos são indicados como procedimentos anestésicos adequados para a realização de mielografias com administração subaracnóidea de iohexol em cães.

\section{AGRADECIMENTOS}

Os autores agradecem à Fundação de Amparo à Pesquisa do Estado de São Paulo (FAPESP), pelo apoio financeiro concedido.

\section{FONTES DE AQUISIÇÃO}

a DIPRIVAN - ZENECA Farmacêutica do Brasil Ltda. - São Paulo, SP, Brasil.

b OHMEDA - mod. Excel 210SE - Datex Ohmeda - Madison, EUA.

c FARS 600 - Lifemed Pesquisas Médicas Ind. e Com. Ltda. São Paulo, SP, Brasil.

d OMNIPAQUE - Sanofi-Synthelabo Ltda. - Rio de Janeiro, RJ, Brasil.

e SEVORANE - ABBOTT Laboratórios do Brasil Ltda, São Paulo, SP, Brasil.
${ }^{\mathrm{f}}$ OHMEDA - mod. 5250 RGM - Datex Ohmeda - Madison, EUA.

${ }^{\mathrm{g}}$ TEB - mod. ECGPC software versão 1.10 - São Paulo, SP, Brasil.

${ }^{\text {h }}$ DIXTAL - mod. DX 2010 - Módulo de PA invasiva - Manaus, AM, Brasil.

DIXTAL - mod. DX 2010 - Módulo de Débito Cardíaco Manaus, AM, Brasil.

\section{REFERÊNCIAS}

ADAMS, W.M. Myelography. Veterinary Clinics of North America: Small Animal Practice, Philadelphia, v.12, n.2, p.295-311, 1982.

AGUIAR, A.J.A. et al. Anestesia por infusão contínua com propofol em cães após medicação pré-anestésica com levomepromazina. Journal of Veterinary Anaesthesia, Newmarket, v.20, p.26-28, 1993.

ALMEIDA, E.M.P. et al. Efeitos cardiocirculatórios da associação de tiletamina/zolazepam em cães (Canis familiaris) pré-tratados ou não pela acepromazina. Brazilian Journal of Veterinary Research and Animal Science, São Paulo, v.37, n.3, p.210-215, 2000.

BRANSON, K.R. et al. A multisite case report on the clinical use of sevoflurane in dogs. Journal of the American Animal Hospital Association, Lakewood, v.37, n.5, p.420-432, 2001.

CHARLES, E.S.; FALLON, W.F. Sevoflurane mask anesthesia for urgent tracheostomy in an uncooperative trauma patient with a difficult airway. Canadian Journal of Anesthesia, Ontario, v.47, n.3, p.242-245, 2000.

CORTOPASSI, S.R.G. Anestesia pediátrica. In: FANTONI, D.T.; CORTOPASSI, S.R.G. Anestesia em cães e gatos. São Paulo: Roca, 2002. Cap.21, p.215-221.

CRYSTAL, G.J. et al. Direct coronary vasomotor effects of sevoflurane and desflurane in situ canine hearts. Anesthesiology, Philadelphia, v.92, n.4, p.1103-1113, 2000.

DENEUCHE, A.; DESBOIS, C. Propofol 2 - Indications and Contra-indications. Point Veterinaire, Maison Alfort, v.30, n.201, p.35-40, 1999.

EBERT, T.J. et al. Cardiovascular responses to sevoflurane, a review. Anesthesia \& Analgesia, Baltimore, v.81, n.6, p.11S-22S, 1995.

FANTONI, D.T. Anestesia no cardiopata. In: FANTONI, D.T.; CORTOPASSI, S.R.G. Anestesia em cães e gatos. São Paulo: Roca, 2002. Cap.30, p.294-320.

GLOWASKI, M.M.; WETMORE, L.A. Propofol: application in veterinary sedation and anesthesia. Clinical Techniques in Small Animal Practice, Philadelphia, v.14, n.1, p.1-9, 1999.

HANOUZ, J.L. et al. In vitro effects of desflurane, sevoflurane, isoflurane and halothane in isolated human right atria. Anesthesiology, Philadelphia, v.92, n.1, p.116, 2000.

KAWATA, R. et al. Changes in cerebral blood flow velocity elicited by surgical stimulation are dependent on the $\mathrm{PaCO}_{2}$ 
level. Canadian Journal of Anesthesia, Ontario, v.48, n.10, p.1029-1033, 2001.

KEEGAN, R.D.; GREENE, S.A. Cardiovascular effects of a continous two hour propofol infusion in dogs comparison with isoflurane anaesthesia. Veterinary Surgery, Philadelphia, v.22, n.6, p.537-543, 1993.

LEITE, A.V. et al. Anestesia para mielografia em cães. Ciência Rural, Santa Maria, v.32, n.4, p.725-729, 2002.

LEWIS, D.D.; HOSGOOD, G. Complications associated with the use of iohexol for myelography of the cervical vertebral column in dogs: 66 cases (1988-1990). Journal of the American Veterinary Medical Association, Schaumburg, v.200, n.9, p.1381-1384, 1992.

MUIR III, W.W.; GADAWSKI, J.E. Respiratory depression and apnea induced by propofol in dogs. American Journal of Veterinary Research, Chicago, v.59, n.2, p.157-161, 1998.

MUTOH, T. et al. Cardiopulmonary effects of sevoflurane, compared with halothane, enflurane, and isoflurane, in dogs. American Journal of Veterinary Research, Chicago, v.58, n.8, p.885-890, 1997.

NUNES, N. Monitoração da anestesia. In: FANTONI, D.T.; CORTOPASSI, S.R.G. Anestesia em cães e gatos. São Paulo: Roca, 2002. Cap. 6, p.64-81.

OLIVA, V.N.L.S. Anestesia Inalatória. In: FANTONI, D.T.; CORTOPASSI, S.R.G. Anestesia em cães e gatos. São Paulo: Roca, 2002. Cap.16, p.174-183.
OMOIGUI, S. Manual de drogas usadas em anestesia. 2.ed. São Paulo: Santos, 1998. 566p.

QUANDT, J.E. et al. Cardiorespiratory and anesthetic of propofol and thiopental in dogs. American Journal of Veterinary Research, Chicago, v.59, n.9, p.1137-1143, 1998.

SHORT, C.E.; BUFALARI, A. Propofol anesthesia. Veterinary Clinics of North America Small Animal Practice, Philadelphia, v.29, n.3, p.747-778, 1999.

SIMON, J.W.; NICHOLAS, J.H.S. Auxílio diagnóstico. In: Diagnóstico e tratamento cirúrgico das afecções espinais do cão e do gato. São Paulo: Manole, 1999. Cap.4, p.34-56.

TACKE, S. et al. Sevoflurane as an inhalation agent in dogs comparison with halothane and isoflurane. Tieraerztliche Praxis Ausgabe K, Kleintiere/Heimtiere, v.26, n.6, p.369377, 1998.

WHITWAM, J.G. et al. The effects of propofol on heart rate, arterial pressure and $\mathrm{A}$ and $\mathrm{C}$ somatosympathetic reflexes in anesthetized dogs. European Journal of Anaesthesiology, Oxford, v.17, n.1, p.57-63, 2000.

WIDMER, W.R. et al. Iohexol and iopamidol myelography in the dog: a clinical trial comparing adverse effects and myelographic quality. Veterinary Radiology \& Ultrasound, Raleigh, v.33, n.6, p.327333, 1992. 